\title{
Uso de Álcool entre Estudantes de Medicina da Universidade Federal do Maranhão
}

\author{
Alcohol Consumption Among Medical \\ Students at the Federal University of \\ Maranhão, Brazil
}

\author{
Felipe Lacerda Barbosa \\ Raphael Lacerda Barbosa \\ Maria do Carmo Lacerda Barbosa ${ }^{I}$ \\ Daniel Lucena de Aguiar \\ Ivan Abreu Figueiredo \\ Antônio Carlos Ribeiro ${ }^{I I}$ \\ Igor Tobias Costa de Castro
}

\author{
PALAVRAS-CHAVE: \\ - Estudantes de Medicina; \\ - Alcoolismo; \\ - Consumo de Bebidas \\ Alcoólicas.
}

\section{KEYWORDS:}

- Students, Medical;

- Alcoholism;

- Alcohol Drinkig.

Recebido em: 27/02/2012

Reencaminhado em: 07/09/2012

Aprovado em: 26/02/2013

REVISTA BRASLLEIRA DE EDUCAÇ̄̃O MÉDICA

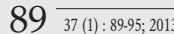

${ }^{I}$ Clinics Hospital of the State University of Campinas, Faculty of Medical Sciences, Campinas, São Paulo, Brazil

II Institute for Education in Medicine and Health, Campinas, SP, Brazil

\section{RESUMO}

Este trabalho avalia o uso de álcool entre estudantes de Medicina da Universidade Federal do Maranhão (UFMA), São Luís, em 2010. Os estudantes responderam dois questionários autoaplicáveis: o primeiro continha informações gerais, e o segundo, o teste Audit, que rastreia consumo de risco. A pesquisa envolveu 337 estudantes, $54,8 \%$ do sexo masculino e $45,2 \%$ do sexo feminino. Duzentos e dezessete $(64,2 \%)$ usavam bebidas alcoólicas. A situação considerada mais propícia para beber foram as festas de faculdade. A maioria dos etilistas (55,8\%) encontrou-se na Zona I pelo escore do Audit (baixo risco para consumo de álcool); 38,2\% na Zona II (médio risco); 4,6\% na Zona III (alto risco); e 1,4\% na Zona IV (altíssimo risco). Houve maior consumo de álcool entre os estudantes de períodos mais adiantados e entre aqueles que não residiam com os pais, com valores de p estatisticamente significantes. É necessário, portanto, orientar o estudante de Medicina sobre os riscos do consumo de álcool de forma nociva e as consequências que este hábito pode trazer para sua profissão.

This study aims to evaluate alcohol consumption among medical students at the Federal University of Maranhão (UFMA), São Luís, Brazil, in 2010. Students completed two self-reported questionnaires: the first containing general information to be collected; and the second an AUDIT test, tracking the risks of their consumption. The research involved 337 students: $54.8 \%$ male and $45.2 \%$ female. Two hundred and seventeen (64.2\%) reported consuming alcohol. The situations considered most suitable for drinking were college parties. Most of the drinkers (55.8\%) fell within Zone I according to the AUDIT score (low-risk alcohol consumption); $38.2 \%$ were in Zone II (medium risk); $4.6 \%$ in Zone III (high risk); and 1,4\% in Zone IV (very high risk). It was found that alcohol consumption was higher among students who were further advanced in their course and among those who did not live with their parents, who showed statistically significant $p$ values. Medical students should therefore be educated on the detrimental risks of alcohol consumption and the consequences that these may entail for their profession. 


\section{INTRODUÇÃO}

O uso moderado de bebidas alcoólicas é considerado um comportamento normal na grande maioria dos países, apesar do consumo excessivo ser menos aceito socialmente. $\mathrm{O}$ álcool é uma das drogas mais utilizadas no Brasil, chegando a ser consumida por quase $70 \%$ da população geral ${ }^{1}$. Além disso, é o principal responsável por acidentes de maior gravidade e mortes violentas ${ }^{2}$.

Desde meados dos anos 1980, o Brasil vem realizando diversos estudos sobre o consumo de álcool e drogas em populações específicas, como estudantes de Medicina ${ }^{3-6}$. Em São Paulo, segundo Kerr-Corrêa et al. ${ }^{7}$, a prevalência do uso de álcool na vida de alunos de Medicina de Botucatu (Unesp) foi de $84 \%$, uma porcentagem bastante alta7.

$\mathrm{O}$ excessivo consumo de álcool entre estudantes de Medicina é um fato preocupante, não só pelos danos pessoais que pode causar, como pelo prejuízo no desenvolvimento e na estruturação de habilidades cognitivo-comportamentais e emocionais, danos ao patrimônio público e violência ${ }^{10-16}$. Além disso, possui impacto negativo em sua atuação como futuros médicos. Sabe-se que o médico influencia direta e indiretamente a saúde pública por meio da habilidade de fazer diagnóstico precoce e tratar, além de servir de modelo para a sociedade $^{17}$

As razões para o abuso de álcool entre estudantes de Medicina são, muitas vezes, o estresse da educação médica ${ }^{18}$ e o fato de a universidade, muitas vezes, ser a primeira experiência do estudante em ser parte de um grupo sem a supervisão dos pais. Esses fatores tornam os estudantes mais vulneráveis a ter experiências ilícitas ou proibidas anteriormente ${ }^{19,20}$. A maioria consegue se adaptar a tal situação, mas, para os que não conseguem, esse conflito pode resultar em depressão, ansiedade, dificuldades acadêmicas, problemas familiares ou abuso de substâncias como o álcool ${ }^{22,23}$. Um dos motivos para o alcoolismo entre futuros médicos é a falha, durante a formação acadêmica, em ensinar o estudante a conhecer seus próprios problemas, assim como os de seus pacientes ${ }^{24}$.

O presente estudo visa avaliar o uso de álcool entre estudantes de Medicina da Universidade Federal do Maranhão.

\section{METODOLOGIA}

Trata-se de um estudo transversal, que analisou o uso de álcool entre estudantes de Medicina da Universidade Federal do Maranhão. A população do estudo foi uma amostra de 338 estudantes distribuídos entre o primeiro e o décimo primeiro períodos do curso de Medicina da UFMA, regularmente matriculados em 2010. Os estudantes do décimo segundo período foram excluídos da pesquisa, em virtude das dificuldades de aplicação do questionário, uma vez que se encontravam em atividades de conclusão do curso.

Foi utilizado um questionário autoaplicável, dividido em duas partes. Na primeira, foram solicitadas informações sobre: sexo, idade, estado civil, semestre do curso, renda familiar, consumo ou não de bebidas alcoólicas, situações mais propícias ao uso de álcool, tipo de bebida alcoólica consumida e uso ou não de tabaco.

Na segunda parte, foi aplicado o Teste para Identificação de Problemas Relacionados ao Álcool (Audit), Versão Autoaplicá$v e l^{26,27,28}$, que é um questionário composto por dez questões a respeito do uso de álcool nos últimos 12 meses. Essa escala é um instrumento desenvolvido pela OMS com a finalidade de identificar pessoas com consumo de risco, uso nocivo e dependência de álcool e avalia o consumo desta substância nos últimos 12 meses. É um teste que tem sido extensivamente testado nos serviços primários de saúde, na população geral, em estudantes universitários e em adolescentes. A aplicação não requer treinamento especializado ${ }^{29}$.

As três primeiras questões fazem parte do domínio que verifica o uso perigoso de álcool, como a quantidade e a frequência do uso de álcool; as três questões seguintes investigam sintomas relacionados à dependência; e as quatro questões finais referem-se ao uso prejudicial de álcool. O escore Audit classifica os indivíduos por níveis de risco em Zona I (0 - 7 pontos), Zona II (8 - 15 pontos), Zona III (16 - 19 pontos) e Zona IV (20 - 40), com aumento progressivo dos riscos tanto maiores sejam as pontuações.

Os dados foram analisados por meio do aplicativo SPSS (Statistical Package for the Social Sciences - Inc. Chicago, Illinois), versão 16.0, e do Bioestat, versão 5.0, utilizando-se o teste do qui-quadrado e o teste $\mathrm{G}$, com nível de significância de 5\%.

Foi realizado um estudo piloto com 24 alunos regularmente matriculados no nono e décimo períodos no primeiro semestre do ano letivo de 2010.

A pesquisa foi aprovada pelo Comitê de Ética em Pesquisa do Hospital Universitário da Universidade Federal do Maranhão, sob o parecer $n^{\circ} 185 / 10$, registro $n^{\circ} 045 / 10$, seguindo as exigências estabelecidas pela Resolução 196 de 1996 do Conselho Nacional de Saúde, que trata da pesquisa com seres humanos.

\section{RESULTADOS}

Do total de estudantes do curso de Medicina da UFMA regularmente matriculados durante o período da pesquisa, conseguiu-se uma amostra de 338, correspondendo a $63 \%$ do alunado. Destes, 185 (54,8\%) eram do sexo masculino e 152 (45,2\%) do sexo feminino, sendo que um estudante foi excluído por não ter respondido corretamente ao questionário (Tabela 1). 
Nessa amostra, observou-se que 217 estudantes (64,2\%) faziam uso de bebidas alcoólicas. Quando analisado o consumo em cada gênero, 58,68\% dos homens apresentaram hábito de beber, enquanto $52,1 \%$ das mulheres tinham o hábito. Realizada análise estatística, o valor de $p$ não foi significante para as diferenças encontradas entre os sexos. Assim, não existe associação entre gênero e o hábito de ingerir bebidas alcoólicas.

Quanto ao estado civil dos entrevistados e ao hábito de beber, $73,0 \%$ dos solteiros, $58,3 \%$ dos casados e $75,0 \%$ dos que tinham relação estável consumiam bebidas alcoólicas. $\mathrm{O}$ teste do qui-quadrado aplicado encontrou um valor de $p=0,5174$, ou seja, não existe associação entre estado civil e o hábito de ingerir bebidas alcoólicas.

TABELA 1

\begin{tabular}{cccc}
\hline & \multicolumn{2}{c}{ Hábito de beber } & valor de $p$ \\
& Sim & Não & \\
\hline Sexo (gênero) & & & \\
\hline Masculino & $123(58,68 \%)$ & $62(52,1 \%)$ & $\mathrm{p}=0,4485$ \\
Feminino & $94(41,32 \%)$ & $57(47,9 \%)$ & \\
Total & $217(100,0 \%)$ & $119(100 \%)$ & \\
\hline
\end{tabular}

\section{Estado civil}

\begin{tabular}{|c|c|c|c|}
\hline Solteiro & $192(88,47 \%)$ & $71(86,58 \%)$ & $p=0,5174$ \\
\hline Casado & $7(3,24 \%)$ & $5(6,11 \%)$ & \\
\hline Relação estável & $18(8,29 \%)$ & $6(7,31 \%)$ & \\
\hline otal & $217(100,0 \%)$ & $82(100,0 \%)$ & \\
\hline
\end{tabular}

\begin{tabular}{cccc}
\hline Renda familiar & & & \\
\hline $1-3 \mathrm{SM}$ & $12(5,73 \%)$ & $11(14,31 \%)$ & $p=0,0717$ \\
$4-6 \mathrm{SM}$ & $44(20,95 \%)$ & $19(24,67 \%)$ & \\
$7-9 \mathrm{SM}$ & $46(21,90 \%)$ & $16(20,77 \%)$ & \\
$>10 \mathrm{SM}$ & $108(51,42 \%)$ & $31(40,25 \%)$ & \\
Total & $210(100,0 \%)$ & $77(100,0 \%)$ & \\
\hline
\end{tabular}

\begin{tabular}{|c|c|c|c|}
\hline \multicolumn{4}{|l|}{ Moradia } \\
\hline Com os pais & $137(59,3 \%)$ & $94(40,7 \%)$ & $p=0,0076$ \\
\hline Com amigos & $22(84,6 \%)$ & $4(15,4 \%)$ & \\
\hline Sozinho & $28(77,8 \%)$ & $8(22,2 \%)$ & \\
\hline Pensionato / república & $7(87,5 \%)$ & $1(12,5 \%)$ & \\
\hline Total & $194(64,5 \%)$ & $107(35,5 \%)$ & \\
\hline
\end{tabular}

Em relação à renda familiar e ao uso de álcool por parte dos estudantes, a pesquisa mostrou que o consumo de álcool entre os que tinham renda familiar de 1-3 salários mínimos (SM) era de 52,2\%, de 4-6 SM era de 69,8\%, de 7-9 SM, 74,2\%, e acima de $10 \mathrm{SM}, 77,7 \%$. O teste do qui-quadrado aplicado encontrou o valor de $p=0,0717$, portanto sem significância estatística. Logo, aceita-se que não existe associação entre renda e o hábito de ingerir bebidas alcoólicas.

Quando analisados a moradia do estudante de Medicina e o hábito de ingerir bebidas alcoólicas, o valor de $p$ foi estatisticamente significante. Encontrou-se o valor de $p$ $=0,0076$ pelo teste do qui-quadrado e o valor de $p=0,0044$ pelo teste G (likelihood ratio). Logo, aceita-se a hipótese alternativa $(\mathrm{H} 1)$ de que existe associação entre moradia e o hábito de beber.

O tipo de bebida mais consumido entre aqueles que fazem uso de álcool foi a cerveja/chope, com 78,34\% de preferência (Tabela 2). Em seguida, vodca $(59,44 \%)$, vinho $(43,31 \%)$, uísque $(31,79 \%)$ e cachaça $(25,34 \%)$. Outras preferências corresponderam a $13,36 \%$. Os entrevistados puderam marcar mais de uma opção neste quesito, havendo alguns que não tiveram uma única preferência.

\section{TABELA 2}

\begin{tabular}{lc}
\hline \multicolumn{1}{c}{ Tipo de bebida consumida } & Hábito de beber \% \\
\hline Cerveja/chope & 78,34 \\
Vodca & 59,44 \\
Cachaça & 25,34 \\
Uísque & 31,79 \\
Vinho & 43,31 \\
Outros & 13,36 \\
\hline
\end{tabular}

Duzentos e cinquenta e sete dos estudantes entrevistados responderam adequadamente quando perguntados sobre a situação mais propícia para beber. A situação mais apontada como propícia foram as festas de faculdade, com $43,6 \%$. Sete por cento marcaram após as provas e 1,6\% ao final de um dia estressante. Os que marcaram como propícias todas as situações anteriores totalizaram $22,2 \%$, e os que em nenhuma das situações anteriormente citadas, $25,7 \%$. A Tabela 3 expõe estes resultados.

Duzentos e noventa e dois assinalaram serem não fumantes $(96,1 \%) ; 8(2,6 \%)$, ex-fumantes; e somente $4(1,3 \%)$ eram 
fumantes, totalizando uma amostra de 304 estudantes. Duzentos e setenta e cinco responderam se fumavam quando bebiam, sendo que 88,3\% marcaram "não"; 8,4\%, "às vezes"; e 3,3\%, "sim".

TABELA 3

Situação mais propícia para beber

\begin{tabular}{lcc}
\hline & $\mathbf{n}$ & $\%$ \\
\hline Festas de faculdade & 112 & 43,6 \\
Após as provas & 18 & 7 \\
Ao final de um dia estressante & 41,6 & \\
$\begin{array}{l}\text { Em todas as situações } \\
\text { anteriormente citadas }\end{array}$ & 57 & 22,2 \\
$\begin{array}{l}\text { Em nenhuma das situações } \\
\text { anteriormente citadas }\end{array}$ & 66 & 25,7 \\
Total & 257 & 100 \\
\hline
\end{tabular}

Analisando a relação do escore Audit com o consumo de bebidas alcoólicas, o teste do qui-quadrado aplicado encontrou o valor de $p<0,0001$. O teste $\mathrm{G}$ (likelihood ratio) encontrou o valor de $p<0,0001$. Em cada teste verifica-se que o valor de $p$ encontrado foi bem menor do que 0,05 , sendo considerado altamente significativo. A partir disso, aceita-se que existe associação entre os pontos do escore Audit e o hábito de ingerir bebidas alcoólicas. Este resultado encontra-se na Tabela 4.

TABELA 4

\begin{tabular}{lcc}
\hline \multirow{2}{*}{ Pontos Audit } & \multicolumn{2}{c}{ Hábito de beber } \\
\cline { 2 - 3 } & \multicolumn{1}{c}{ Sim } & Não \\
\hline Zona I & $121(55,8 \%)$ & $120(100 \%)$ \\
Zona II & $83(38,2 \%)$ & $0(0,0 \%)$ \\
Zona III & $10(4,6 \%)$ & $0(0,0 \%)$ \\
Zona IV & $3(1,4 \%)$ & $0(0,0 \%)$ \\
Total & $217(100 \%)$ & $120(100 \%)$ \\
\hline
\end{tabular}

$p<0,0001 ;$ significância se $p<0,05$

Com relação ao semestre cursado, 92,1\% dos alunos do nono semestre apresentaram o hábito de consumir bebidas alcoólicas, representando a classe com maior prevalência de uso de álcool dentre os semestres avaliados. Aplicando o teste do qui-quadrado para verificar associação entre a variável e a ingestão alcoólica, encontrou-se o valor de $p=0,0029$. Assim, aceita-se que existe associação entre o semestre cursado e o hábito de beber, como apresentado na Tabela 5 .

TABELA 5

\begin{tabular}{|c|c|c|}
\hline \multirow{2}{*}{$\begin{array}{l}\text { Semestre } \\
\text { cursado }\end{array}$} & \multicolumn{2}{|c|}{ Hábito de beber } \\
\hline & Sim & Não \\
\hline $1^{\mathrm{o}}$ & $19(47,5 \%)$ & $21(52,5 \%)$ \\
\hline $2^{\underline{o}}$ & $16(61,5 \%)$ & $10(38,5 \%)$ \\
\hline $3^{\circ}$ & $27(67,5 \%)$ & $13(32,5 \%)$ \\
\hline $4^{\mathrm{o}}$ & $17(56,7 \%)$ & $13(43,3 \%)$ \\
\hline $5^{\mathrm{o}}$ & $14(45,2 \%)$ & $17(54,8 \%)$ \\
\hline $6^{\mathrm{o}}$ & $16(69,6 \%)$ & $7(30,4 \%)$ \\
\hline $7^{\circ}$ & $20(60,6 \%)$ & $13(39,4 \%)$ \\
\hline $8^{\circ}$ & $18(72,0 \%)$ & $7(28,0 \%)$ \\
\hline $9^{\circ}$ & $35(92,1 \%)$ & $3(7,9 \%)$ \\
\hline $10^{\circ}$ & $20(64,5 \%)$ & $11(35,5 \%)$ \\
\hline $11^{\circ}$ & $15(78,9 \%)$ & $4(21,1 \%)$ \\
\hline Total & $182(61,1 \%)$ & $116(38,9 \%)$ \\
\hline
\end{tabular}

$p=0,029 ;$ significância se $p<0,05$.

\section{DISCUSSÃO}

A amostra avaliada neste estudo foi significante, pois correspondeu a $63 \%$ dos estudantes do curso de Medicina da UFMA regularmente matriculados no segundo semestre do ano de 2010, do primeiro ao décimo primeiro períodos.

O percentual de etilistas foi alto, totalizando $64,4 \%$. Pesquisas realizadas entre estudantes de Medicina da Universidade Federal de Uberlândia e entre sextanistas da Universidade de FreeState, na África do Sul, mostram resultados semelhantes, sendo $66,34 \%$ e $85,2 \%$, respectivamente ${ }^{21,25}$.

$\mathrm{O}$ encontro de consumo também elevado de bebidas alcoólicas no sexo feminino é motivo de preocupação, já que é bem documentada na literatura médica a maior suscetibilidade desse gênero aos efeitos lesivos do álcool. A mulher tem menor concentração de álcool-desidrogenase gástrica e menor volume de distribuição corporal. Além disso, os fatores hormonais próprios da mulher a tornam mais propensa à doença hepática alcoólica ${ }^{30}$. A maioria dos estudos, entretanto, mostra o consumo maior entre os homens ${ }^{7,9,21,25}$. 
Em nosso estudo, a cerveja e o chope foram as bebidas de maior preferência entre os entrevistados, fato também verificado por Paduani et al..$^{25}$ em pesquisa realizada entre acadêmicos de Medicina da Universidade Federal de Uberlândia (UFU). De acordo com o Levantamento Nacional sobre Padrões de Consumo de Álcool na População Brasileira ${ }^{32}$, 60\% da população do nosso país tem preferência por este tipo de bebida ${ }^{31}$.

De maneira semelhante às pesquisas realizadas em outras escolas médicas ${ }^{7,9}$, nosso estudo não constatou relação estatisticamente significativa entre o hábito de beber e a condição de estar solteiro, apesar de estes constituírem a maioria dos entrevistados (88\%). Esta característica está provavelmente relacionada ao fato de o curso de Medicina exigir muitos anos de formação e, por conseguinte, um longo caminho até se atingir a independência profissional ${ }^{33}$.

Na UFMA, o etilismo também não teve relação com a renda familiar. Todavia, é interessante atentar para a informação de que praticamente metade $(48,43 \%)$ dos estudantes tem renda familiar acima de dez salários mínimos, e 21,6\% de 7 a 9 salários (Tabela 1). Pode-se inferir que o curso de Medicina da UFMA é composto, em sua maioria, pela classe média alta da sociedade, que tem maior condição de acesso a informação e conhecimento. Kerr-Corrêa et al. ${ }^{7}$ mostraram que na Unesp $40 \%$ das famílias tinham renda superior a 20 salários mínimos/mês. No entanto, afirmam que nem sempre o aluno se sente à vontade para falar de renda familiar?

A literatura é enfática ao enfocar situações que podem induzir ao uso de álcool, como as festas de faculdade e os próprios fatores estressores inerentes à prática médica ${ }^{8,16}$. De maneira semelhante, no presente estudo, as festas de faculdade foram as situações citadas como as mais propícias para se beber. No entanto, aparece em destaque a opção "em nenhuma das opções anteriormente citadas" com o segundo maior percentual $(25,7 \%)$, fato que levanta curiosidade para maior investigação, a fim de conhecer estas outras situações.

O número de tabagistas entre os estudantes de Medicina da UFMA foi baixo. Somente $1,2 \%$ se declararam fumantes, e 2,6\%, ex-fumantes. Um estudo realizado com 300 acadêmicos do curso de Medicina da Universidade Luterana Brasileira (Ulbra) em 2005, no Rio Grande do Sul, apontou prevalência de $18,6 \%$ de fumantes e 7,3\% de ex-fumantes ${ }^{34}$. Apesar de ocorrerem essas disparidades entre as diversas faculdades de Medicina, o percentual de tabagistas entre graduandos do curso médico é menor do que a população geral brasileira ${ }^{35}$.

Além de avaliar o consumo de álcool e sua associação com diversas variáveis, a pesquisa avaliou o consumo de risco de bebidas alcoólicas por meio da aplicação do questionário $\mathrm{Al}$ - cohol Use Disorders Identification Test (Audit). A Organização Mundial de Saúde (OMS) desenvolveu e adotou o Audit por ser um questionário simples, de dez questões, rapidamente aplicável, com o intuito de facilitar aos médicos o rastreamento de casos de consumo de risco na rotina diária ${ }^{28}$.

Em nossos resultados, conforme a Tabela 4, mais da metade se enquadra na Zona I (menos que 8 pontos), ou seja, bebem com padrão de baixo risco ou não bebem. As pessoas que estão nesta faixa devem apenas ser informadas sobre as consequências do consumo de risco ${ }^{28}$. No entanto, $44,2 \%$ estão nas Zonas II, III e IV, tendo um padrão considerado potencialmente danoso à saúde. Este resultado reflete a necessidade urgente de desenvolver políticas públicas de educação para a saúde não só no curso de Medicina, mas também entre os profissionais de saúde.

Resultado interessante também encontrado em nosso estudo foi quanto à associação entre semestre cursado e uso de álcool. Outros artigos publicados sobre o tema também apontam essa associação: a de que existe maior uso dessa substância com o decorrer da graduação $0^{7,9,9,25}$. Com o curso de Medicina da UFMA, como já referido, não foi diferente. Verificamos que as turmas, com o passar do tempo, tendem a ter um número crescente de etilistas.

Um dos dados do questionário envolvia o quesito "moradia", no qual o menor consumo de álcool foi encontrado nos estudantes que moravam com os pais. Estudantes que moram com os pais têm tendência a não beber ${ }^{25}$ por, talvez, existir maior controle e rigidez sobre suas atitudes.

Por fim, é importante relatar as limitações encontradas no trabalho, de modo que novas pesquisas possam reconhecê-las e superá-las. Sendo um estudo transversal, os resultados encontrados informam sobre a situação do consumo de álcool em um momento particular, ou seja, não há o seguimento dos indivíduos de modo a identificar os efeitos a longo prazo. Com relação ao teste Audit, sendo este autoaplicável, devem-se levar em conta as possíveis dificuldades de compreensão das perguntas do questionário e, principalmente, respeitar a função específica do teste, que se limita ao rastreamento do consumo de risco para o álcool.

\section{CONCLUSÃO}

Detectou-se que o curso de Medicina da Universidade Federal do Maranhão tem uma taxa alta de etilistas $(64,2 \%)$, sem diferenças estatisticamente significativas do hábito de beber entre os sexos. A maioria dos estudantes é solteira e de classe média, sendo que o estado civil e a renda familiar não influenciaram no consumo de álcool. Informação importante foi observada em relação ao tabagismo, que é baixíssimo neste 
estudo $(1,2 \%)$, e em relação à moradia, que foi relevante fator influenciador do hábito de beber.

Apesar do grande número de etilistas, constatou-se que mais da metade dos acadêmicos (55,8\%) estão na Zona I do escore Audit. Entretanto, existe um percentual significativo $(38,2 \%)$ na Zona II. Deve-se dar atenção a este dado, já que a tendência, sem intervenção, é de migrarem para as zonas seguintes. Além disso, existe a tendência de crescer o número de etilistas a cada período do curso, demonstrando que as turmas mais próximas da conclusão têm um número maior de alunos que usam bebidas alcoólicas. Esse é um fato interessante, pois se pode inferir que existem estudantes que iniciam a graduação sem beber e terminam a faculdade etilistas.

\section{CONSIDERAÇÕES FINAIS}

A pesquisa, com suas limitações, aponta a necessidade de políticas educacionais no curso de Medicina da UFMA que proporcionem ao estudante uma profunda reflexão sobre o consumo de bebidas alcoólicas e as consequências que esse hábito pode trazer ao dia a dia do futuro médico. Uma vez que essa não é uma falha exclusiva da UFMA, mas de praticamente todas as escolas médicas brasileiras, é necessário maior foco sobre o tema.

\section{REFERÊNCIAS}

1. Galduróz JCF, Caetano R. Epidemiology of alcohol use in Brazil. Rev Bras Psiquiatr. 2004; 26(supl.2): 3-4.

2. Ferreira MP, Laranjeira R. Dependência de substâncias psicoativas. In: Ito L, org. Terapia cognitivo-comportamental para transtornos psiquiátricos. Porto Alegre: Artes Médicas; 1998.

3. Andrade AG, Bassit AZ, Mesquita AM, Fukushim JT, Gonçalves EL, et al. Prevalência do uso de drogas entre alunos da Faculdade de Medicina da Universidade de São Paulo (1991-1993). Rev ABP-APAL. 1995;17:41-6

4. Andrade AG, Bassit AZ, Zerr-Corrêa F, Aparecido TA, Eduardo Paulo B, Myrian C, et al. Fatores de risco associados ao uso de álcool e drogas na vida, entre estudantes de medicina do Estado de São Paulo. Rev ABP-APAL. 1997; 19:117-126.

5. Cruz AR, Cardoso JD. Avaliação do consumo e indução ao abuso de drogas entre alunos do curso de medicina. Ciênc Cultura. 1988;40:935.

6. Mesquita AMC, Bucaretchi HA, Castel S, Andrade AG. Estudantes da Faculdade de Medicina da Universidade de São Paulo: uso de substâncias psicoativas em 1991. Rev. ABP-APAL. 1995;17:47-54.
7. Kerr-Corrêa F, Andrade AG, Bassit AZ, Boccuto NMVF Uso de álcool e drogas por estudantes de medicina da Unesp. Rev Bras Psiquiatr. 1999;21;95-100.

8. Lemos KM, Neves NMBC, Yoichi Kuwano A, Tedesqui G, Bitencourt AGV, Neves FBCS, et al. Uso de substâncias psicoativas entre estudantes de Medicina de Salvador (BA). Rev Psiq Clín. 2007;35:118-24.

9. Marais AL, Calitz FJW, Rataemane LUZ, Joubert G et al. Alcohol use among sixth-year medical students at the University of the Free State. South African J Psych. 2002; 8:79-84.

10. Chassin L, Pitts SC, Prost J. Binge drinking trajectories from adolescent to emerging adulthood in a high-risk sample: Predictors a substance abuse outcomes. J Consult Clin Psychol. 2002;70:67-78.

11. O'malley PM, Johnston LD. Epidemiology of alcohol and other drug use among American college students. Studies Alcohol.2002;14:23-9.

12. Perkins HW. Surveying the damage: a review of research on consequences of alcohol misuse in college populations. Studies Alcohol. 2002;14:91-100.

13. Zeigler DW, Wang CC, Yoast RA, Dickinson BD, McCaffree MA, Robinowitz CB, Sterling MC. The neurocognitive effects of alcohol on adolescents and college students. Prev Med. 2005;40:23-32.

14. Ham LS, Hope DA. College students and problematic drinking: A review of the literature. Clin Psychol Rev. 2003;23:719-59.

15. Park CL, Grant C. Determinants of positive e negative consequences of alcohol consumption in college students: alcohol use, gender e psychological characteristics. Addictive Beh. 2005;30:755-65.

16. Windle M. Alcohol use among adolescents and young adults. Alcohol Res Health.2003;27:79-85.

17. Gray JD, Bhopal RS, White M. Developing a medical school alcohol policy. Med Educ. 1998;32:138-42.

18. Guthrie EA, Black D, Shaw CM, Hamiton J, Creed FH, Tomenson B. Embarking upon a medicalcareer: psychological morbidity in first-year medical students. Med Educ.1995;29:337-41.

19. Walsh A. Drug use and sexual behavior: users, experimenters and abstainers. J Soc Psychol. 1992; 132:691-3.

20. Leibsohn JM. Relationship between drug and alcohol use and peer group association of college freshmen as they transit from high school. J Drug Education.1994; 24:177-92.

21. Marais AL, Calitz FJW, Rataemane LUZ, Joubert G. Alcohol use among sixth-year medical students at the University of the Free State. South African J of Psych. 2002;8:79-84. 
22. Aktekin M, Karaman T, Senol YY, Erdem S, Erengin H, Akaydin M. Anxiety, depression and stressful life events among medical students: a prospective study in Antalya, Turkey. Med Educ. 2001; 35:12-7.

23. Edwards M, Zimet C. Problems and concerns among medical students. J Med Educ. 1975; 51:619-25.

24. Ashton $\mathrm{CH}$, Kamali F. Personality, lifestyles, alcohol and drug consumption in a sample of British medical students. Med Educ. 1995;29:187-92.

25. Paduani GF, Barbosa GA, Morais JCR, Pereira JCP, Almeida FM. Consumo de álcool e fumo entre os estudantes da Faculdade de Medicina da Universidade Federal de Uberlândia. Rev Bras Educ Med. 2008;32:66-74.

26. Babor TF, Higgins-Biddle JC, Saunders JB, Monteiro MG. The Alcohol Use Disorders Identification Test: Guidelines for use in primary care. Geneva; WHO; 2001.

27. Babor TF, Higgins-Biddle JC, Saunders JB, Monteiro MG. AUDIT: teste para identificação de problemas relacionados ao uso de álcool: roteiro para uso em atenção primária. Ribeirão Preto: PAI-PAD; 2003.

28. Furtado EF, Yosetake LL. Coisas simples que todo médico pode fazer para tratar o alcoolismo. Você já faz?.Rev. Medical Sigma Pharma. 2005;1:13-7.

29. Segatto ML, Souza e Silva R, Laranjeira R, Pinsky I. O impacto do uso de álcool em pacientes admitidos em umpronto-socorro geral universitário. Rev Psiquiatr Clín. 2008;35:138-43.

30. Lopes AC. Tratado de Clínica Médica; 2006.

31. Pinsky I A. Propaganda de Bebidas Alcoólicas no Brasil [online]. 2006. Disponível em: <http:/ / www.propagandasembebida.org.br/artigos/integra.php?id=12>

32. Secretaria Nacional Antidrogas. Gabinete de Segurança Nacional. I Levantamento Nacional sobre os Padrões de Consumo de Álcool na População Brasileira. Brasília; 2007.
33. Lourenção LG, Moscardini AC, Soler ZASG. Saúde e Qualidade de Vida de Médicos Residentes. Rev Assoc Med Bras. 2010;56:91.

34. Zettler EW, Nudelmann LM, Cunha DP, Hilgert C, Mattos M, Scholl M et al. Prevalência do tabagismo entre estudantes de Medicina e fatores de risco associados. Revista AMRIGS. 2005; 49:16-19.

35. Ministério da Saúde. Instituto Nacional de Câncer. Programa Nacional de Controle do Tabagismo e Outros Fatores de Risco de Câncer. Modelo Lógico e Avaliação. 2ª ed. Rio de Janeiro; 2003.

\section{CONTRIBUIÇÃO DOS AUTORES}

Felipe Lacerda Barbosa: Concepção e desenho do estudo, coleta de dados, análise dos resultados e confecção do manuscrito. Raphael Lacerda Barbosa: Coleta e análise dos dados, análise crítica do conteúdo Maria do Carmo Lacerda Barbosa: Auxílio na análise dos dados, revisão crítica do conteúdo intelectual Daniel Lucena de Aguiar: Coleta de dados e participação do desenho do estudo Ivan Figueiredo: Orientador do trabalho e análise crítica do conteúdo intelectual Antônio Carlos Ribeiro: Análise estatística e revisão dos dados Igor Tobias: Coleta de dados e participou do desenho do estudo.

\section{CONFLITO DE INTERESSES}

Declarou não haver.

\section{ENDEREÇO PARA CORRESPONDÊNCIA}

Rua Imperatriz, Quadra $13-\mathrm{n}^{\mathrm{o}} 08$

Jardim Eldorado - Turu - São Luís

CEP 65066-300 MA

E-mail: fl_barbosa@hotmail.com. 